\title{
Bioavailability assessment of topical delivery systems: in vitro delivery of minoxidil from prototypical semi-solid formulations
}

\author{
Chia-Ming Chiang ${ }^{1}$, G.L. Flynn ${ }^{1}$, N.D. Weiner ${ }^{1}$, W.J. Addicks ${ }^{1}$ and G.J. Szpunar ${ }^{2}$ \\ ${ }^{1}$ College of Pharmacy, University of Michigan, Ann Arbor, MI 48109 (U.S.A.) and ${ }^{2}$ The Upjohn Company, Kalamazoo, MI (U.S.A.)
}

(Received 9 February 1988)

(Revised version received 3 June 1988)

(Accepted 27 June 1988)

Key words: Topical delivery; Vehicle; Thin Application; Diffusion

\begin{abstract}
Summary
An in vitro technique has been developed for evaluating the delivery performance of topical semi-solid formulations. A thin and uniform layer of formulation was applied in facsimile to actual usage conditions by troweling the vehicle across a thin, circular copper template ( $200 \mu \mathrm{m}$ in thickness). Approximately $30-45 \mathrm{mg}$ of an oil-in-water cream, a water-in-oil cream or an ointment, each containing a range of concentrations of minoxidil, were applied over human cadaver skin within a defined circular area of $1.54 \mathrm{~cm}^{2}$. The rates of permeation of minoxidil from these formulations were determined by finite dose diffusion experiments. For formulations containing $2 \%$ minoxidil, the flux from the w/o cream tested was, about 4 times higher than fluxes from the $o / w$ cream and the ointment. Even though all $\mathrm{w} / \mathrm{o}$ formulations were initially saturated with drug, the flux of minoxidil from these creams increased as the concentration of minoxidil was increased from $0.5 \%$ to $2 \%$. In contrast, the delivery rates from the o/w cream and the ointment did not appear to be dependent on the minoxidil concentration applied $(0.5-2 \%)$. Under the operative experimental conditions, the percent coefficients of variation of flux of minoxidil from these formulations were less than $20 \%$. To achieve this low level of variability, the skin samples were all obtained from the same cadaver abdomen. If one assumes that the efficacy of a particular formulation is dependent on the ability of the drug to be released from the vehicle and diffuse through the skin, the studies show that the nature of the vehicle can profoundly affect delivery even when excess solid drug is present. They also indicate that reliable in vitro comparisons of drug delivery are possible as long as one performs the studies on skin samples taken from the same section of skin.
\end{abstract}

\section{Introduction}

There is an increasing emphasis on the transdermal delivery of drugs to treat systemic diseases. However, the majority of drugs used for topical administration are intended to treat local skin disorders. Certainly for systemic action, and in all likelihood for topical action as well, the efficacy of a particular formulation is dependent on the abil-

Correspondence: N.D. Weiner, College of Pharmacy, University of Michigan, Ann Arbor, MI 48109, U.S.A. ity of the drug to be released from the vehicle and diffuse through the skin and especially its outermost layer, the stratum corneum *. The thermodynamic and kinetic determinants of delivery here are complex and attempts to evaluate the effecti-

\footnotetext{
* The exact relationship between flux through the skin and delivery into the local tissues of the skin is substantially unresolved. Certainly when flux is high there are steeper concentration gradients across the living tissue. It is presumed by us that these correlate with higher local concentrations and greater efficacy at the local level, but this remains unproven.
} 
veness of various formulations by in vitro methods have generally been unsuccessful. One problem is that there is no well developed experimental technique to apply finite doses as thin films in facsimile to the usage situation. Thus, although the necessity of having an in vitro method to correlate with the in vivo studies is recognized, there is no simple and generally accepted technique to sort out the pcrformance of formulations effectively (Guy et al. 1986).

The purpose of this study is to demonstrate that the relative drug delivery merits of topical, semi-solid dosage forms can be sorted out using in vitro diffusion cell techniques as has been conjectured earlier by Addicks et al. (1987) based on studies involving synthetic membranes. This approach involves the application of a thin and uniform film of formulation to human cadaver skin and determination of fluxes of a given drug by finite dose diffusion experiments. The test compound chosen for the studies was minoxidil, which has been shown to be of some benefit in the treatment of alopecia areata when applied topically (Weiss et al., 1981). The procedures were devcloped as part of our mission to cstablish a standard method to evaluate the performance of semi-solid topical formulations.

\section{Materials and Methods}

\section{Materials}

Minoxidil, USP milled, was supplied by The Upjohn Co. (Kalamazoo, MI). The [piperidine${ }^{3} \mathrm{H}$ ]minoxidil was obtained from the same source with a radiochemical purity of $98 \%$ and a spec. act. of $25 \mathrm{Ci} / \mathrm{mmol}$. The various vehicle components, namely, mineral oil (Mallinkrodt, Paris, KY) Carbopol 940 (BF Goodrich, Cleveland, $\mathrm{OH}$ ). Quadrol polyol (BASF Corp., Chemical Div., Parsippany, NJ), Tween 60, Arlacel 60, Arlacel 83 (ICI Americas, Wilmington, DE), white petrolatum (CMC, Smyrna, TN) and white wax were used as received.

\section{Preparation of minoxidil formulations}

A recrystallized mixture of radiolabelled and cold minoxidil was prepared by the following pro-
TABLE 1

Formulae for each of the minoxidil-containing vehicles tested

\begin{tabular}{lccc}
\hline Component & \multicolumn{3}{l}{ Parts by weight } \\
\cline { 2 - 4 } & o/w Cream & w/o Cream & Ointment \\
\hline White petrolatum & - & 45 & 50 \\
White wax & - & 2 & 5 \\
Mineral oil & 20 & - & 45 \\
Tween 60 & 2.8 & - & - \\
Arlacel 60 & 2.2 & 1 & - \\
Arlacel 83 & - & 3 & - \\
Water & 75 & 50 & - \\
Carbopol 940 & 0.3 & - & - \\
Quadrol & q.s. & - & - \\
\hline
\end{tabular}

cedure. $\left[{ }^{3} \mathrm{H}\right]$ minoxidil stock solution in methanol (about $0.3 \mathrm{mCi}$ in $300 \mu \mathrm{l}$ ) was placed in a $100 \mathrm{ml}$ flask and dried under nitrogen. The dried radiolabelled minoxidil was mixed together with about $100 \mathrm{mg}$ of unlabeled minoxidil and $4 \mathrm{ml}$ warm ethanol were added to dissolve the mixture. About $80 \mathrm{ml}$ hexane were then added to initiate recrystallization of the minoxidil. The flask was chilled in an ice bath for $4 \mathrm{~h}$ and the recrystallized minoxidil powder was collected under vacuum and placed in a desiccator overnight to facilitate removal of the solvents. The specific activity of the recrystallized minoxidil was determined to be $4-5.6 \mu \mathrm{Ci} / \mathrm{mg}$. Since the amount of radiolabelled minoxidil available was limited, $0.5 \%, 1 \%$ and $2 \%$ minoxidil formulations were prepared by incorporating a suitable amount of the recrystallized minoxidil into approximately $400 \mathrm{mg}$ of each of three vehicles (Table 1) contained on a watch glass with the aid of a small pestle.

\section{Application of formulation}

To establish the technique for applying thin reproducible films of each formulation onto the skin, the following was done. First, a piece of Parafilm (American Can Co., Greenwich, CT) with size of about $4 \mathrm{~cm}^{2}$ was cut and weighed. A template $(200 \mu \mathrm{m}$ thick, brass disc of diameter 3.1 $\mathrm{cm}$ with a hole of diameter $1.4 \mathrm{~cm}$ cut in the center) was then put on top of the Parafilm. The formulation was applied to the surface of the Parafilm and troweled flush with the edge of the template with the aid of a cover glass. The tem- 
plate was carefully removed and the Parafilm containing the formulation was again weighed. The net weight of the formulation applied on the Parafilm was determined from the difference between the two measurements. An evaluation of the technique for applying thin films to human cadaver skin was also done.

To prepare the skin membrane, skin samples (about $200 \mu \mathrm{m}$ in thickness) were taken with a dermatome from a single cadaver donor. These were placed in the freezing compartment of a standard laboratory refrigerator and frozen at the temperature of the compartment; $-20^{\circ} \mathrm{C}$. Tissue was kept frozen at this temperature until an experiment was begun. Portions of the frozen skin were thawed before use and appropriate-sized sections of the skin $\left(4 \mathrm{~cm}^{2}\right)$ were cut and put on a larger piece of Parafilm with the stratum corneum facing upward. The weight of the skin sample was then determined. The residual dermis was not separated from the dermatomed skin sample since preliminary results had shown that the flux of minoxidil through the separated epidermis was no different than through the dermatomed skin. The formulation was applied to the surface of the skin as described and the weight was measured. The net weight of the formulation applied on the skin was found from the difference between the two weight measurements.

\section{Permeation studies}

The abdominal skin of a deceased 85 years old white female was used for the permeation experiments. After the minoxidil formulation was applied to the skin membrane with formulation facing upward, the tissue was mounted in a Franz diffusion cell (Crown Glass, Somerville, NJ). Formulation was applied to a skin section and the section was mounted in a diffusion cell in one continuous operation; only a minute or two elapsed from the actual application to initation of a run. The receiver compartment was maintained at $37^{\circ} \mathrm{C}$ with circulating water. The reservoir contained phosphate buffer ( $\mathrm{pH} 7$ ) and was stirred with a bar magnet. The phosphate buffer was equilibrated to $37^{\circ} \mathrm{C}$ before the start of the experiments and the receiver compartment was partially filled with the buffer. After assembling the diffusion cells, phosphate buffer was added to the receiver compartment to a predetermined level.

The moment of mounting the membrane on a Franz cell was defined as the "zero time" for each run. At predetermined time intervals samples were withdrawn from the receiver compartment by a 1 $\mathrm{ml}$ tuberculin syringe with a 23-gauge needle fitted with a section of PE-50 polyethylene tubing. Each volume removed for sampling was replaced with an equal volume of phosphate buffer. Exactly 0.5 $\mathrm{ml}$ of each sample were pipetted into $10 \mathrm{ml}$ liquid scintillation fluid (Safety Solve, RPI, Mount Prospect, IL) and assayed on a scintillation counter.

\section{Analysis of data}

After correcting concentrations for dilution associated with repetitive sampling, the concentration data were plotted as the cumulative amount collected in the receiver compartment as a function of time. The flux of minoxidil per unit area of membrane into the reveiving reservoir in the quasi-steady state is equal to:

$J=\frac{V}{A} \cdot \frac{\mathrm{d} C}{\mathrm{~d} t}$

where $J$ is the per unit area pseudo-steady-state flux $\left(\mathrm{mg} / \mathrm{cm}^{2} / \mathrm{h}\right)$ across the skin; $A$ is the area for diffusion $\left(\mathrm{cm}^{2}\right) ; V$ is the volume of the receiver compartment $(\mathrm{ml})$; and $\mathrm{dC} / \mathrm{d} t$ is the steady-state slope $(\mathrm{mg} / \mathrm{ml} / \mathrm{h})$.

\section{Results}

The weights for the applications of the 3 formulations and the reproducibility resulting from the procedure used to apply the formulations as uniform thin films over Parafilm are indicated in Table 2. The average weights of applications over human cadaver skin concur with those placed over Parafilm (Table 2), essentially showing that the nature of the membrane presented no major difficulties in spreading the films. Moreover, it was found that the creams and ointment could be applied to either of these surfaces reproducibly, with the percent coefficient of variation generally less than $15 \%$. This was considered adequate re- 
TABLE 2

Weight of formulations applied on Parafilm and on human cadaver skin with a copper template $11.54 \mathrm{~cm}^{2}$ in area and 200 $\mu m$ in thickness

\begin{tabular}{llll}
\hline Sample no. & \multicolumn{3}{l}{ Weight (mg) applied on parafilm } \\
\cline { 2 - 4 } & w/o Cream & o/w Cream & Ointment \\
\hline 1 & 40.3 & 26.8 & 24.7 \\
2 & 53.3 & 38.0 & 37.3 \\
3 & 45.8 & 36.5 & 33.7 \\
4 & 42.8 & 30.9 & 30.5 \\
5 & 41.0 & 30.0 & 28.5 \\
6 & 40.8 & 29.6 & 27.7 \\
7 & - & 21.7 & 24.4 \\
8 & - & 21.1 & - \\
9 & 51.8 & 36.5 & 37.3 \\
10 & 43.2 & 31.1 & 31.4 \\
11 & 41.9 & 30.1 & 30.2 \\
Avg. \pm S.D. & $44.5 \pm 4.8$ & $30.2 \pm 5.5$ & $30.6 \pm 4.5$ \\
\cline { 2 - 4 } & Weight (mg) applied on human cadaver skin \\
\cline { 2 - 4 } 1 & 48.6 & - & 31.4 \\
2 & 50.0 & - & 31.6 \\
3 & 40.5 & - & 26.1 \\
4 & 37.5 & - & 25.6 \\
5 & 48.5 & - & 26.7 \\
6 & 35.4 & - & 22.9 \\
7 & - & & $26.6 \pm 3.7$ \\
Avg. \pm S.D. & $43.4 \pm 6.4$ & & \\
\hline & & & \\
\hline
\end{tabular}

producibility for the research purposes. Representative plots of fluxes of $2 \%$ of minoxidil from the various formulations tested are shown in Fig. 1. The fluxes of minoxidil as a function of minoxidil concentration from the 3 formulations are summarized in Table 3.

\section{Discussion}

In vitro methods involving determination of the rate and extent of penetration of an applied drug through the skin are often employed to assess topical drug delivery. Altough many approaches have been tried, there is no generally accepted technique to evaluate the kinetics of delivery of drugs from dermatological formulations. Rather, the majority of studies associated with the development of topical drugs have dealt with the innate permeation of a given drug or a family of analogs. Most have not recognized and addressed the fact that interactions which modulate drug delivery exist between the drug, its vehicle and the skin. As a result, formulation effects are generally not well understood. Furthermore, while many studies are conducted that employ synthetic membranes or animal skin membranes as model systems to predict the in vivo clinical situation, there is some evidence that vehicle-skin and drug-skin interactions are species-dependent and human skin responds in ways unpredictable from studies involving animal skin tissue and "model membranes". Finally, existing in vitro techniques generally fail to deal realistically with the amount of topical media actually applied to the skin surface.

The in vitro technique described here is our first attempt to evolve a method for evaluating the in vivo performance of semi-solid formulations for topical drug delivery. Human cadaver skin prepared in thin sheets with the aid of a dermatome was used to avoid species-dependent effects on delivery. To eliminate high inter-subject and intra-subject skin variations reported by other research groups (Langguth et al., 1986; Southwell et al., 1984), an abdominal section from a single human cadaver was used for all experiments.

Since the thickness of a formulation theoretically affects the release of a drug from a given formulation (Flynn et al., 1985; Guy and Hadgraft, 1980), thin layers of the formulations were applied to the skin instead of infinitely thick slabs. This was achieved by using a $200 \mu \mathrm{m}$ thick copper

\section{TABLE 3}

Fluxes of minoxidil from different formulations through human cadaver skin determined by finite dose experiments. The diffusional area of the skin for all the experiments was $1.54 \mathrm{~cm}^{2}$

Values given are mean \pm S.D. with number of experiments given in parentheses.

\begin{tabular}{llll}
\hline $\begin{array}{l}\text { Minoxidil } \\
\text { conc. }\end{array}$ & \multicolumn{3}{l}{ Minoxidil flux $\left(\mathrm{mg} / \mathrm{h} / \mathrm{cm}^{2} \times 10^{4}\right)$} \\
$\%(\mathrm{w} / \mathrm{w})$ & $\mathrm{w} / \mathrm{o}$ Cream & $\mathrm{o} / \mathrm{w}$ Cream & Ointment \\
\hline 0.5 & & & \\
1.0 & $1.4 \pm 0.1(3)$ & $0.40 \pm 0.10(3)$ & $0.75 \pm 0.13(3)$ \\
2.0 & $2.1 \pm 0.3(3)$ & $0.34 \pm 0.04(3)$ & - \\
& $2.7 \pm 0.3(6)$ & $0.53 \pm 0.05(4)$ & $0.65 \pm 0.08(4)$ \\
\hline
\end{tabular}




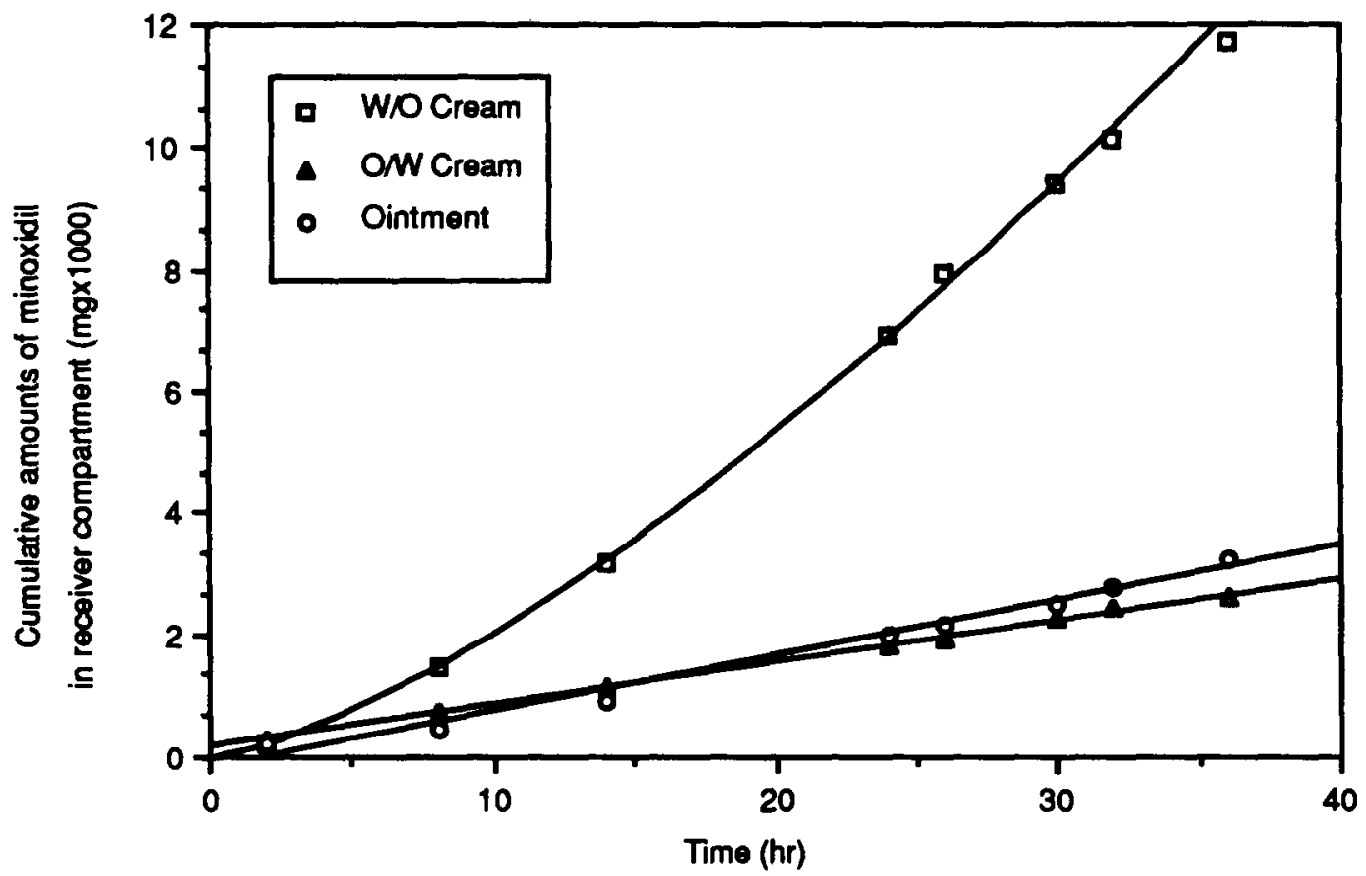

Fig. 1. Representative plots of permeation of $2 \%$ minoxidil from various vehicles through human cadaver skin by finite dose experiments. The diffusional area of the skin for all the experiments was $1.54 \mathrm{~cm}^{2}$.

template as a mold. Troweling the formulation across the template results in highly reproducible films (Table 2) with coefficients of variation of the weights of these applications typically being $10-15 \%$. Based on the density of each formulation $(0.85,0.83,0.89$ for ointment, $w / o$ cream and $\mathrm{o} / \mathrm{w}$ cream, respectively), the applied thickness of ointment, w/o cream and $o / w$ cream are estimated as $234 \pm 35,348 \pm 38$ and $223 \pm 62 \mu \mathrm{m}$, respectively. The estimated thicknesses of the $\mathrm{o} / \mathrm{w}$ cream and ointment film relate well to the thickness of the template $(200 \mu \mathrm{m})$ while the estimated thickness of $w / o$ cream appears half again thicker than the depth of the well of the template. We are unable to offer a satisfying reason why the latter films weigh $50 \%$ more than anticipated.

Representative plots of permeation of minoxidil from the $2 \% \mathrm{o} / \mathrm{w}$ cream, the $\mathrm{w} / \mathrm{o}$ cream and the ointment (Fig. 1) indicate that minoxidil accumulates at a steady rate in the receiver compartment after an initial lag period. The achievement of steady-state was not unexpected since minoxidil was present in each of the formulations in amounts exceeding its solubility. The slope of the steadystate line was used to calculate flux values of minoxidil from the various formulations.

An examiniation of Table 2 points out two important effects of the formulation on the flux of minoxidil through the skin. Firstly, the w/o cream is clearly the superior formulation with respect to minoxidil transport. It delivers more minoxidil per unit time (even at $0.5 \%$ ) than the other two formulations. Whereas this is apparent at all minoxidil concentrations tested, it is most evident at the $2 \%$ concentration level $(P<0.01)$. Of considerable further interest, the $\mathrm{w} / \mathrm{o}$ formulation showed a significant concentration dependence with respect to the delivery of minoxidil (e.g. $P<0.01$ for the $2 \%$ cream vs the $0.5 \%$ cream). Minoxidil is present as suspended matter at each of the concentrations tested and thus there is no reason to suspect that this formula is affecting the barrier properties of the skin in some proportion to the amount of minoxidil applied. Therefore, the concentration dependency appears to be a clear demonstration of a circumstance where the kinetics of solubiliza- 
tion and diffusion in the vehicle have a major influence on the rate of delivery. This concentration dependence could be due to more rapid dissolution of drug from the higher concentration formulations as a result of greater surface area of the suspended particulates. Exactly how the evaporation of the internal water phase affects the kinetics is obscure but it may be a contributing factor. Perhaps more important than the individual differences in the delivery from the formulations in this study is the fact that such differences can in fact be demonstrated when great care is taken to define the experimental system and protocol used.

\section{Ackowledgements}

This study was supported by The Upjohn Co., Kalamazoo, MI and by Grant AI/GM22303 from the National Institutes of Health.

\section{References}

Addicks, W.J., Flynn, G.L. and Weiner, N., Validation of a flow-through diffusion cell for use in transdermal research. Pharm. Res., 4(1987) 337-341.

Flynn, G.L., Topp, E.M. and Amidon, G.L., Physicochemical aspects of drug delivery to and via the skin. In Topics in Pharmaceutical Sciences, Elsevier, Amsterdam, 1985, pp 313-328.

Guy, R.H., Guy, A.H., Maibach, H.l. and Shah, V.P., The bioavailability of dermatological and other topically administered drugs. Pharm. Res., 3 (1986) 253-262.

Guy, R. and Hadgraft, J., A theoretical description relating skin penetration to the thickness of the applied medicament. Int. J. Pharm, 6 (1980) 321-332.

Langguth, P., Spahn, H., Mutschler, E. and Hubner, K., An approach to reduce the number of skin samples in testing the transdermal permeation of drugs. J. Pharm. Pharmacol., 38 (1986) 726-730.

Southwell, D., Barry, B.W. and Woodford, R., Variations in permeability of human skin within and between specimens. Int. J. Pharm., 18 (1984) 299-309.

Wess, V.C., West, D.P. and Mueller, C.E., Topical minoxidil in alopecia areata. I. Am. Acad. Dermatol., 5 (1981) 224-226. 